\title{
AVALIAÇÃO DE LAVAGEM PARCIAL DE SAIS EM NEOSSOLO FLÚVICO IRRIGADO, UTILIZANDO MODELAGEM COMPUTACIONAL ${ }^{1}$
}

\author{
ADRIANO L. N. MONTEIRO ${ }^{2}$, ABELARDO A. DE A. MONTENEGRO ${ }^{3}$, \\ SUZANA M. G. MONTENEGRO ${ }^{4}$, FRANCISCO X. DOS SANTOS ${ }^{2}$
}

\begin{abstract}
RESUMO: Este trabalho foi realizado em um vale aluvial irrigado do semiárido do Brasil (Estado de Pernambuco). O objetivo foi investigar o efeito de lâminas de lixiviação e de precipitação no controle da salinidade do solo em lisímetros de drenagem. Com base em simulações numéricas, utilizando-se do modelo HYDRUS-1D, avaliaram-se o fluxo e o transporte de sais na zona não saturada. Mediante medidas de campo de evapotranspiração real e precipitação, foi possível inferir que o modelo se apresentou como ferramenta adequada nas simulações realizadas, para diferentes cenários de lâminas de lixiviação. Verificou-se, experimental e numericamente, que a lixiviação parcial pode ser utilizada como alternativa de manejo para a redução da salinidade na zona radicular, desde que precipitações efetivas possam complementar o processo de lavagem.
\end{abstract}

PALAVRAS-CHAVE: semiárido, salinidade, aquífero aluvial.

\section{PARTIAL SALT LEACHING EVALUATION IN AN IRRIGATED FLUVISSOL THROUGH COMPUTATIONAL MODELING}

\begin{abstract}
This study has been developed in an irrigated alluvial aquifer in the semi-arid zone of Brazil (State of Pernambuco). The objective was to investigate the effect of different leaching depths and precipitation on reducing soil salinity in drainage lysimeters. Based on numeric simulations using the HYDRUS-1D model, flow and salt transport in the unsaturated zone were evaluated. By using measured actual evapotranspiration and precipitation, it was verified that the model is a satisfactory tool to simulate flow and transport in the studied situations considering different leaching depths. Numerical and experimental results pointed out that the partial leaching could be used for reduction of the salinity in the root zone, as far as effective precipitation might complement the leaching process.
\end{abstract}

KEYWORDS: semiarid, salinity, alluvial aquifer.

\section{INTRODUÇÃO}

A região semiárida do Nordeste brasileiro possui a agricultura como um dos principais elementos da sua economia. Em especial, a agricultura familiar constitui-se em uma das principais componentes na produção de alimentos. Contudo, a região sofre limitações para a prática da agricultura familiar em virtude da instabilidade climática, como o regime irregular de chuvas e a elevada taxa de evaporação (SANTIAGO et al., 2004), bem como a reduzida disponibilidade de recursos hídricos, tanto em termos quantitativos quanto qualitativos. Devido aos escassos recursos hídricos superficiais, as águas subterrâneas são frequentemente utilizadas na agricultura irrigada.

A salinidade da água subterrânea de aquíferos aluviais, explorada por Poços Amazonas para a irrigação na região, conforme AUDRY \& SUASSUNA (1995), varia de 0,14 dS m $\mathrm{d}^{-1}$ a $3,80 \mathrm{dS} \mathrm{m}^{-1}$. MONTENEGRO et al. (2001) caracterizaram as águas subterrâneas de aluvião no Agreste do Estado de Pernambuco, que apresenta clima semiárido, e encontraram valores de condutividade elétrica de $0,36 \mathrm{dS} \mathrm{m}^{-1}$ a 11,75 $\mathrm{dS} \mathrm{m}^{-1}$, com média de $1,47 \mathrm{dS} \mathrm{m}^{-1}$, avaliada a partir de 972 amostras.

\footnotetext{
${ }^{1}$ Extraído da tese de doutorado do primeiro autor.

${ }^{2}$ Eng $^{\text {o }}$ Agrônomo, Doutor em Agronomia, Programa de Pós-Graduação em Agronomia - Ciência do Solo, UFRPE, Recife - PE, Fone (0XX81) 3320.6220,ad_normandia@ @otmail.com

${ }^{3}$ Eng $^{\mathrm{o}}$ Civil, Prof. Adjunto, Departamento de Tecnologia Rural, UFRPE, Recife - PE.

${ }^{4}$ Eng ${ }^{\circ}$ Civil Prof. Adjunto, Departamento de Engenharia Civil, UFPE, Recife - PE.

Recebido pelo Conselho Editorial em: 13-9-2007
}

Aprovado pelo Conselho Editorial em: 21-4-2009

Eng. Agríc., Jaboticabal, v.29, n.2, p.207-220, abr./jun. 2009 
A utilização de águas com qualidade inferior, ou seja, de moderada a alta salinidade na irrigação e sem apropriada lixiviação de sais, pode promover a salinização do solo, prejudicando o desenvolvimento radicular dos cultivos. De acordo com RHOADES (1974), a necessidade de lixiviação de sais pode ser calculada a partir da salinidade da água de irrigação e da salinidade tolerada pela cultura. Entretanto, em períodos com ocorrência de chuvas, a necessidade de lixiviação teórica pode ser reduzida, devido ao efeito de lavagem natural promovido pelas precipitações. Em tais situações, poder-se-ia realizar apenas uma lixiviação parcial dos sais por meio da lâmina de irrigação.

O manejo da salinidade na agricultura irrigada depende, dentre outros fatores, da estimativa precisa dos termos de balanço hídrico na zona radicular. Dentre as metodologias de avaliação dos balanços de água e sais na zona não saturada, destacam-se os lisímetros, que permitem determinar a evapotranspiração real de uma cultura em desenvolvimento, de referência, ou para determinar a evaporação do solo nu, segundo ABOUKHALED et al. (1982), além da contabilização dos termos de drenagem do perfil com precisão (SILVA et al., 2003).

Complementarmente, a modelagem matemática também permite a estimativa de balanços de água e sais, e está sendo cada vez mais utilizada no estabelecimento de políticas protetoras do ambiente. Vários modelos foram desenvolvidos para a simulação determinística e estocástica de fluxo de água e de transporte de solutos. Quando não se consideram as incertezas dos parâmetros físicos, tem-se a modelagem determinística, enquanto a modelagem estocástica se caracteriza por incluir distribuições estatísticas dos parâmetros (MONTENEGRO et al., 2002).

MONTENEGRO et al. (2002) aplicaram modelagem determinística e estocástica ao modelo WAVE (VANCLOOSTER et al., 1994) para avaliar o risco de salinização em vale aluvial, no Agreste de Pernambuco, fornecendo-se o intervalo de confiança de $95 \%$ para a condutividade elétrica de Neossolo irrigado. Embora se tenha verificado que as concentrações de sais simuladas pela formulação determinística se aproximaram daquelas produzidas pela modelagem estocástica, os autores destacam a necessidade de obtenção de mais informações de campo para uma realística simulação de fluxo e de transporte, utilizando-se de metodologia determinística.

GONÇALVES et al. (2006) utilizaram o modelo HYDRUS-1D em trabalho de avaliação de risco de salinização, em três lisímetros, verificando a sua eficiência nas simulações determinísticas de fluxo de água e de transporte de solutos em lisímetros irrigados com água de diferentes qualidades, em um Fluvissolo Eutrófico do Alentejo (Portugal).

Diante disso, conduziu-se este estudo em um vale aluvial suscetível à salinização, sendo o objetivo avaliar o efeito combinado de diferentes lâminas de lixiviação e da precipitação pluviométrica na redução da salinidade do solo, adotando-se lisímetros irrigados com água moderadamente salina e utilizando-se do modelo HYDRUS-1D como ferramenta computacional determinística, calibrado ao experimento de campo.

\section{MATERIAL E MÉTODOS}

A Fazenda Nossa Senhora do Rosário, onde foi realizada esta pesquisa, está localizada em Pesqueira-PE, Agreste Pernambucano, a $228 \mathrm{~km}$ da cidade de Recife. Com área de aproximadamente 110 ha, suas terras distribuem-se em estreita faixa aluvial do Rio Ipanema, com coordenadas geográficas de $08^{\circ} 10^{\prime} 25^{\prime}$ ' $\mathrm{S}, 35^{\circ} 11^{\prime} 25^{\prime}$ ' $\mathrm{W}$, de latitude e longitude, respectivamente, e $650 \mathrm{~m}$ de altitude (RIBEIRO \& CORRÊA, 2001). Segundo MONTENEGRO et al. (2002), a profundidade do lençol freático varia espacial e sazonalmente entre 2,0 e 5,0 m, e a condutividade elétrica da água subterrânea entre 0,6 e 3,1 dS $\mathrm{m}^{-1}$. Neste estudo, utilizou-se água com condutividade média de $1,10 \mathrm{dS} \mathrm{m} \mathrm{m}^{-1}$ e RAS de $6,58\left(\mathrm{mmolc} \mathrm{L}^{-1}\right)^{0,50}$, proveniente do aquífero aluvial.

A área dispõe de um pluviômetro "Ville de Paris", Tanque Classe “A”, estação meteorológica automática e lisímetros. 
Amostras de solo foram coletadas para determinação de parâmetros hidrodinâmicos e caracterização física em laboratório. A textura no perfil do solo foi determinada pelo método do densímetro, enquanto a densidade do solo foi determinada segundo o método do anel volumétrico, e de partículas, pelo método do balão volumétrico (EMBRAPA, 1997).

\section{Montagem do sistema experimental}

O estudo experimental foi conduzido em lisímetros cultivados com cenoura (Daucus carota L.) durante 100 dias. Na semeadura, adotou-se o espaçamento de $20 \mathrm{~cm}$ entre linhas. Vinte dias após o plantio, foi realizado o desbaste, mantendo-se o espaçamento de $5 \mathrm{~cm}$ entre plantas.

O sistema experimental foi composto por três lisímetros de drenagem, denominados de lisímetro 1 (L1), lisímetro 2 (L2) e lisímetro 3 (L3), com características similares às propostas por SANTOS et al. (2008). Os lisímetros foram construídos utilizando-se, para tal, de reservatório em fibra de vidro com capacidade de $1 \mathrm{~m}^{3}$, diâmetro superficial de $1,39 \mathrm{~m}$, diâmetro da base de $1,10 \mathrm{~m}$ e altura de $0,8 \mathrm{~m}$. Os lisímetros foram preenchidos com o solo removido a cada $20 \mathrm{~cm}$, preservando-se as características das camadas originais. Para induzir um sistema de drenagem livre, os últimos $10 \mathrm{~cm}$ do perfil foram preenchidos por brita $\mathrm{n}^{\mathrm{0}} 19$, e adotados tubos de PVC soldável de $25 \mathrm{~mm}$ perfurados, revestidos por manta têxtil. Os lisímetros permitiram a contabilização dos termos do balanço hídrico com base na equação da conservação de massa. Para a medição da umidade e da tensão do extrato da solução do solo, instalou-se em cada lisímetro uma estação tensiométrica com manômetro de mercúrio, cápsulas de extração de solução do solo e tubo de acesso para a sonda de nêutrons.

Previamente ao início do plantio, os lisímetros foram irrigados até à saturação e, em seguida, cobertos até à finalização da drenagem, quando se procedeu ao plantio. Os tensiômetros foram instalados nas profundidades de 10;20;30;40 e $60 \mathrm{~cm}$, tendo sido monitorados diariamente ao longo do período do cultivo. Na Tabela 1, constam os atributos físicos do solo nas profundidades estudadas.

TABELA 1. Caracterização física média do solo dos lisímetros. Average physical characterization of the lysimeters soil.

\begin{tabular}{cccccc}
\hline \multirow{2}{*}{ Profundidade (cm) } & \%areia & \%silte & \%argila & $\begin{array}{c}\text { Massa Específica Aparente Massa Específica Real das } \\
\text { Seca do Solo }\left(\mathrm{g} \mathrm{cm}^{-3}\right)\end{array}$ & \begin{tabular}{c} 
Partículas $\left(\mathrm{g} \mathrm{cm}^{-3}\right)$ \\
\hline $0-20$
\end{tabular} \\
\hline 47,50 & 31,80 & 20,70 & 1,32 & 2,53 \\
$20-40$ & 51,52 & 25,28 & 17,20 & 1,35 & 2,47 \\
$40-60$ & 53,52 & 26,00 & 21,20 & 1,35 & 2,56 \\
\hline
\end{tabular}

\section{Monitoramento das propriedades físicas e químicas nos lisímetros}

Nos lisímetros, foram monitoradas a tensão da água no solo (diariamente), a drenagem do perfil (diariamente), assim como a condutividade elétrica da solução do solo (semanalmente). No período de 79 a 90 dias do início do experimento, as leituras de condutividade elétrica se procederam de dois em dois dias, de modo a monitorar o efeito de eventos pluviométricos extremos.

A coleta da solução do solo foi realizada por bomba de sucção manual conectada a tubos de extração dotados de cápsula porosa. Os extratores de solução foram instalados nas profundidades de $20 \mathrm{~cm}, 30 \mathrm{~cm}, 40 \mathrm{~cm}$ e $60 \mathrm{~cm}$. Aos extratores foi aplicada tensão de $60 \mathrm{kPa}$ e, logo em seguida, realizada leitura nos tensiômetros. Após 24 horas, coletou-se a solução no interior dos tubos. Os valores de condutividade elétrica obtidos na solução do solo por meio dos extratores foram corrigidos para a umidade de saturação, conforme SILVA et al. (2000).

\section{Simulação computacional com o modelo HYDRUS-1D}

O HYDRUS-1D é um modelo numérico que utiliza o método dos elementos finitos para a simulação da dinâmica de água e de soluto em meios porosos (ŠIMŮNEK et al., 2005), com grande 
aplicação na Engenharia de Água e Solo (NEVES et al., 2000). O modelo foi desenvolvido para simular fluxo de água, temperatura e transporte de solutos em meios não saturados e saturados (ŠIMŮNEK et al., 2005), apresentando formulação teórica similar à do modelo WAVE. O HYDRUS-1D apresenta várias vantagens em relação ao modelo WAVE, dentre as quais as interfaces com o usuário, particularmente para a entrada de parâmetros, e de sub-rotinas para a estimativa inversa de parâmetros hidráulicos.

O fluxo no meio poroso é descrito pela equação de Richards para meio não saturado, incorporando o termo (S), sumidouro ou extração de água pela raiz para contemplar a evapotranspiração [eq. (1)]:

$$
\frac{\partial \theta}{\partial \mathrm{t}}=\frac{\partial}{\partial \mathrm{z}}\left[\mathrm{K}(\mathrm{h})\left(\frac{\partial \mathrm{h}}{\partial \mathrm{z}}+1\right)\right]-\mathrm{S}(\mathrm{h})
$$

em que,

$\theta$ - umidade do solo na base de volume, $\mathrm{L}^{3} \mathrm{~L}^{-3}$

$\mathrm{t}$ - tempo, $\mathrm{T}$;

$\mathrm{h}$ - potencial mátrico, L;

$\mathrm{K}(\mathrm{h})$ - condutividade hidráulica, $\mathrm{LT}^{-1}$;

$\mathrm{Z}$ - profundidade, $\mathrm{L}$, e

$\mathrm{S}(\mathrm{h})$ - termo de extração de água pelo sistema radicular $\left(\mathrm{L}^{3} \mathrm{~L}^{-3} \mathrm{~T}^{-1}\right)$.

Para a determinação da extração de água pela raiz, o modelo aplica o método proposto por FEDDES (1978), e modificado por GENUCHTEN (1987) para incluir estresse osmótico, segundo a eq. (2):

$$
\mathrm{S}\left(\mathrm{h}, \mathrm{h}_{\varphi}\right)=\alpha\left(\mathrm{h}, \mathrm{h}_{\varphi}\right) \mathrm{S}_{\text {máx }}
$$

em que,

$\mathrm{S}_{\max }$ - taxa máxima de extração de água, $\left.\mathrm{L}^{3} \mathrm{~L}^{-3} \mathrm{~T}^{-1}\right)$, e

$\alpha(\mathrm{h}, \mathrm{h} \varphi)$ - função de resposta ao potencial mátrico e osmótico.

A redução do rendimento da cultura devido ao potencial osmótico é relacionada linearmente à condutividade elétrica (CE) do extrato de saturação (MAAS \& HOFFMAN, 1977), segundo o modelo proposto por GENUCHTEN (1987).

As funções hidráulicas do solo foram consideradas segundo os modelos de GenuchtenMualem (GENUCHTEN, 1980), relacionando o potencial mátrico do solo com o teor de umidade volumétrica, e expressando-se a condutividade hidráulica em função da umidade volumétrica. As funções são definidas segundo as equações:

$$
\mathrm{S}_{\mathrm{e}}=\frac{\left(\theta-\theta_{\mathrm{r}}\right)}{\left(\theta_{\mathrm{s}}-\theta_{\mathrm{r}}\right)}=\left(1+\alpha|\mathrm{h}|^{\mathrm{n}}\right)^{-\mathrm{m}}
$$

em que,

$\mathrm{S}_{\mathrm{e}}$ - saturação efetiva $\left(0 \leq \mathrm{S}_{\mathrm{e}} \leq 1\right)$;

$\theta_{\mathrm{r}}$ - umidade residual;

$\theta_{\mathrm{s}}$ - umidade na saturação, e

$\alpha, n$ e $m$ - parâmetros de ajuste da curva com os dados experimentais.

O parâmetro empírico de ajuste da curva $m$ é obtido pela relação $m=1-\frac{1}{n}$, proposta por MUALEM (1976).

A função de condutividade hidráulica é escrita como:

$$
\mathrm{K}=\mathrm{K}_{\text {sat }} \mathrm{S}_{\mathrm{e}}^{\mathrm{x}}\left[1-\left(1-\mathrm{S}_{\mathrm{e}}^{\mathrm{x} / \mathrm{m}}\right)^{\mathrm{m}}\right]^{2}
$$

em que, 
x - parâmetro de inclinação (MUALEM, 1976), adotado igual a 0,5, para a maioria dos solos e Ksat é a condutividade hidráulica saturada.

As simulações ao longo do perfil dos lisímetros de drenagem foram realizadas por um período de 100 dias, correspondendo ao ciclo da cultura da cenoura, com os primeiros 50 dias sendo utilizados para a calibração dos parâmetros do modelo de Genuchten (e de transporte), e o período restante, para a validação numérica.

Optou-se por não realizar testes de condutividade hidráulica nos lisímetros para não produzir deformações nas camadas de solo, de modo a evitar o surgimento de caminhos preferenciais de fluxo. Desse modo, os valores dos parâmetros hidráulicos de Genuchten foram estimados a partir de correlações com propriedades texturais da área, considerando o solo dos lisímetros e as caracterizações físicas e hidráulicas procedidas por BASTOS (2004) ao longo do perfil (Tabela 2), e posteriormente calibrados por meio das simulações.

TABELA 2. Parâmetros da curva de retenção de água no solo, encontrados por BASTOS (2004), na área em estudo. Parameters of the water retention curve in soil according to BASTOS (2004) in the studied area.

\begin{tabular}{ccccc}
\hline Parâmetros & $20 \mathrm{~cm}$ & $40 \mathrm{~cm}$ & $60 \mathrm{~cm}$ & $80 \mathrm{~cm}$ \\
\hline \%Areia & 37,05 & 48,52 & 52,66 & \\
\hline $\mathrm{n}$ & 1,70 & 1,43 & 1,28 & 1,18 \\
$\alpha$ & 0,001 & 0,017 & 0,044 & 0,061 \\
$\Theta_{\mathrm{r}}$ & 0,16 & 0,14 & 0,11 & 0,017 \\
$\Theta_{\text {sat }}$ & 0,37 & 0,41 & 0,39 & 0,43 \\
$\mathrm{~K}_{\text {sat }}\left(\mathrm{cm} \mathrm{dia}^{-1}\right)$ & 16,8 & 95,28 & 54,48 & 30,96 \\
\hline
\end{tabular}

\section{Transporte de solutos}

O transporte de solutos no perfil do solo ocorre pela advecção e dispersão na fase líquida, assim como pela difusão na fase gasosa (ŠIMU゚NEK et al., 2005). De acordo com FORKUTSA (2006), o fluxo de massa total em solos irrigados pode ser descrito como a soma do fluxo por advecção e do fluxo por dispersão, negligenciando-se o efeito da difusão. Nesse caso, a equação de advecção-dispersão no HYDRUS-1D é resolvida numericamente, podendo ser expressa por:

$$
\frac{\partial(\theta \mathrm{C})}{\partial \mathrm{t}}=\frac{\partial}{\partial \mathrm{z}}\left(\theta \mathrm{D}_{\mathrm{h}} \frac{\partial \mathrm{C}}{\partial \mathrm{z}}-\mathrm{q}_{\mathrm{z}} \mathrm{C}\right)
$$

em que

$\mathrm{C}$ - concentração do soluto na fase líquida do solo, $\mathrm{M} \mathrm{L}^{-3}$;

$\mathrm{z}$ - coordenada vertical, L;

$\mathrm{D}_{\mathrm{h}}$ - coeficiente de dispersão hidrodinâmica, $\mathrm{L}^{2} \mathrm{~T}^{-1}$, e

$\mathrm{q}_{\mathrm{z}}$ - fluxo de massa, $\mathrm{M} \mathrm{L}^{-2} \mathrm{~T}^{-1}$.

A dispersão hidrodinâmica foi inicializada, para a calibração, com base no coeficiente de dispersividade $\lambda=3 \mathrm{~cm}$, conforme adotado por MONTENEGRO et al. (2002), para solo similar no mesmo vale aluvial. Para as simulações numéricas, os perfis de solo dos lisímetros foram discretizados a cada $10 \mathrm{~cm}$, seguindo as amostragens de campo.

\section{Parâmetros de entrada no modelo}

A condição de contorno superior foi especificada conforme a precipitação, a irrigação e os valores particionados da evapotranspiração em transpiração e evaporação do solo nu. A evapotranspiração de referência foi calculada em função da evaporação do Tanque Classe "A", adotando-se coeficiente de tanque $\mathrm{K}_{\mathrm{p}}=0,75$, de acordo com DOORENBOS \& KASSAM (1979), 
para as condições de bordadura às quais o tanque estava exposto, da umidade relativa do ar e da velocidade do vento local.

Para o desmembramento dos termos potenciais de evaporação do solo $\left(\mathrm{E}_{\mathrm{p}}\right)$ e transpiração $\left(\mathrm{T}_{\mathrm{p}}\right)$, foi adotado o modelo proposto por VANCLOOSTER et al. (1994). O modelo estima a evaporação potencial, em função do índice de área foliar (IAF), e da evapotranspiração potencial da cultura, segundo as expressões:

$$
\begin{aligned}
& \mathrm{Ep}=\mathrm{e}^{-0.6 \mathrm{IAF}} \mathrm{ETc} \\
& \mathrm{Tp}=\mathrm{ETc}-\mathrm{Ep}
\end{aligned}
$$

em que,

$\mathrm{IAF}$ - índice de área foliar;

$\mathrm{E}_{\mathrm{p}}$ - taxa de evaporação potencial, $\mathrm{LT}^{-1}, \mathrm{e}$

$\mathrm{T}_{\mathrm{p}}$ - taxa de transpiração potencial, $\mathrm{LT}^{-1}$.

Neste estudo, foram adotados os valores de índice de área foliar da cenoura apresentados por SUOJALA (2000) e mostrados na Tabela 3.

TABELA 3. Valores de índice de área foliar da cenoura (Fonte: SUOJALA, 2000). Values for the carrot leaf area index (Source: SUOJALA, 2000).

\begin{tabular}{llcccccc}
\hline DAS & 0 & 43 & 55 & 64 & 75 & 85 & 96 \\
IAF & 0 & 0,19 & 0,59 & 1,9 & 4,1 & 5,95 & 5,7 \\
\hline
\end{tabular}

DAS - dia após a semeadura; IAF - índice de área foliar

\section{RESULTADOS E DISCUSSÃO}

As simulações ao longo do perfil dos três lisímetros foram realizadas por um período de 100 dias, durante o ciclo de cenoura, conforme já comentado. Na Figura 1, apresenta-se a distribuição temporal da evapotranspiração real medida em cada lisímetro. Os totais pluviométricos foram registrados tanto manualmente quanto em estação automática, situada a $300 \mathrm{~m}$ de distância da área experimental.

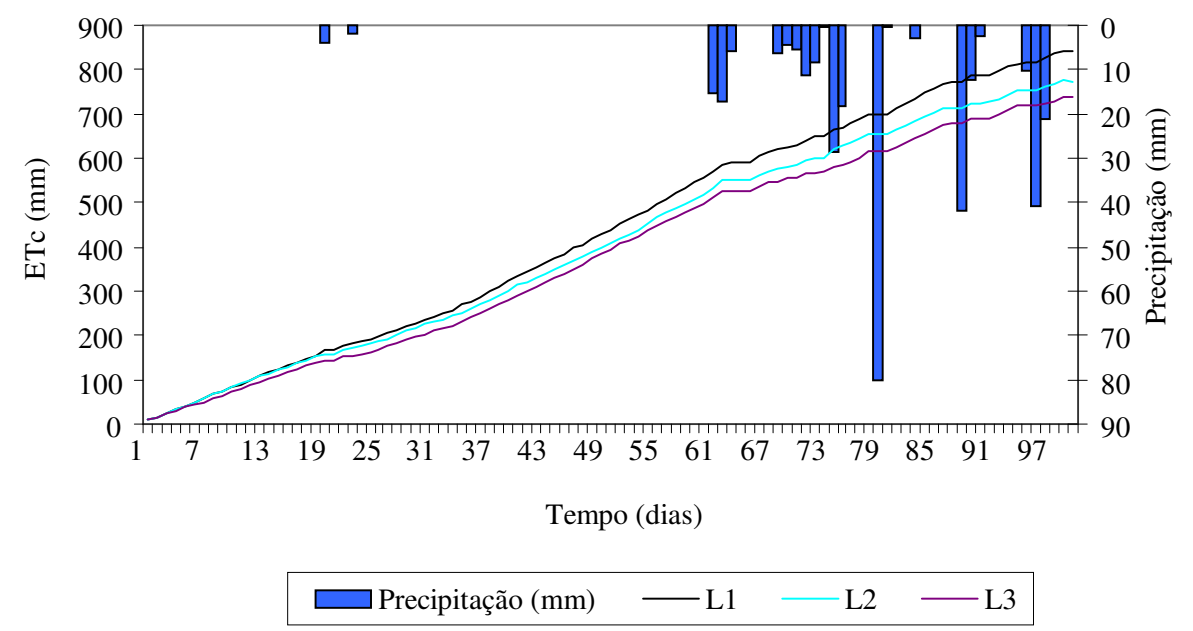

FIGURA 1. Evapotranspiração acumulada nos lisímetros (L) e eventos de precipitação. Cumulative evapotranspiration in the lysimeters $(L)$ and rainfall events.

As frações de lixiviação (FL) experimentais foram: $\mathrm{FL}=0,13$ para o lisímetro $\mathrm{L} 1 ; \mathrm{FL}=0,11$ para o lisímetro $\mathrm{L} 2$, e $\mathrm{FL}=0,20$ para o lisímetro $\mathrm{L} 3$, no período antecedente ao das chuvas (até o dia 62). Tais valores são menores que a necessidade de lixiviação de 0,28 para a produtividade de $100 \%$ da cenoura, segundo o modelo de RHOADES (1974), e com água de irrigação de $1,10 \mathrm{dS} \mathrm{m}^{-1}$. 
Com o intuito de verificar o desenvolvimento da cultura com os manejos adotados, foram realizadas avaliações biométricas da cenoura, conforme apresentado na Tabela 4.

TABELA 4. Parâmetros biométricos da cultura da cenoura, em cada lisímetro. Crop biometric parameters of carrot in each lysimeter.

\begin{tabular}{ccccc}
\hline Lisímetro & Parâmetros Biométricos & Média & Desvio-Padrão & C.V. (\%) \\
\hline \multirow{2}{*}{1} & CR (cm) & 14,22 & 3,23 & 22,69 \\
& D (mm) & 27,53 & 5,55 & 20,15 \\
& Peso (g) & 51,53 & 24,89 & 48,31 \\
\hline \multirow{2}{*}{2} & CR (cm) & 13,19 & 1,90 & 14,40 \\
& D (mm) & 29,94 & 4,42 & 14,78 \\
3 & Peso (g) & 59,92 & 23,83 & 39,78 \\
\hline \multirow{2}{*}{3} & CR (cm) & 12,40 & 2,93 & 23,61 \\
& D (mm) & 24,96 & 4,79 & 19,20 \\
& Peso (g) & 37,83 & 18,41 & 48,68 \\
\hline
\end{tabular}

CR- comprimento da raiz; D- diâmetro medial; C.V. - coeficiente de variação

SANTOS (2008) encontrou os valores médios de 14,80 $( \pm 2,96) \mathrm{cm}$ e $28,03( \pm 4,84) \mathrm{mm}$ para as variáveis comprimento da cenoura e diâmetro medial, respectivamente, para experimento na mesma área, no período de 28-10-2005 a 2-2-2006. Assumindo-se normalidade aos dados, podemse extrapolar intervalos de confiança $(13,69 ; 15,91 \mathrm{~cm})$ e $(26,23 ; 29,84 \mathrm{~mm})$, respectivamente. Desse modo, os comprimentos obtidos para a cultura nos lisímetros L2 e L3 diferem levemente, enquanto os diâmetros mediais para os lisímetros L1 e L2 não diferiram dos encontrados por SANTOS (2008), com leve diferença para o lisímetro L3. De posse do anteriormente exposto, há elementos para se assumir que a cultura se desenvolveu dentro da realidade local de clima semiárido e de água de irrigação moderadamente salina $\left(\mathrm{CE}=1,10 \mathrm{dS} \mathrm{m} \mathrm{m}^{-1}\right)$.

\section{Resultados numéricos}

Duas condições de contorno foram analisadas: drenagem livre e potencial especificado (e variável no tempo). As simulações com drenagem livre não se mostraram pertinentes e tenderam a superestimar o fluxo descendente para todos os lisímetros, consequentemente, subestimando as umidades nos nós da malha. A razão para tais inconsistências foi detectada ao se analisar os potenciais matriciais do solo na base dos lisímetros, via de regra exibindo valores próximos à saturação. Em verdade, tais valores indicam que houve saturações temporárias dos lisímetros.

Desse modo, decidiu-se por adotar condição de contorno inferior de potencial especificado, medido a $80 \mathrm{~cm}$ de profundidade. Tal condição melhorou sobremaneira as simulações, bem como as calibrações.

As calibrações buscaram otimizar os parâmetros hidráulicos e de transporte das diversas camadas dos lisímetros. $\mathrm{Na}$ Tabela 5, apresentam-se os valores calibrados para o experimento.

TABELA 5. Valores de calibração do modelo, para as funções de GENUCHTEN (1987). Calibration values of the model, for the van GENUCHTEN (1987) functions.

\begin{tabular}{ccrcc}
\hline Profundidade das Camadas $(\mathrm{cm})$ & $0-20$ & $20-40$ & $40-70$ & $70-80$ \\
\hline$\%$ Areia & 47,5 & 51,52 & 53,52 & - \\
$\mathrm{n}$ & 1,43 & 1,32 & 1,26 & 1,01 \\
$\alpha$ & 0,019 & 0,03 & 0,05 & 0,04 \\
$\Theta_{\mathrm{r}}$ & 0,12 & 0,10 & 0,09 & 0,045 \\
$\Theta_{\mathrm{s}}$ & 0,40 & 0,41 & 0,42 & 0,43 \\
$\mathrm{~K}_{\mathrm{sat}}\left(\mathrm{cm} \mathrm{dia}^{-1}\right)$ & 42,31 & 87,12 & 63,07 & 94,00 \\
\hline
\end{tabular}


Na Figura 2, apresentam-se as comparações em pentadas (média de 5 dias) para os potenciais mátricos medidos e simulados, nas profundidades de $10 \mathrm{~cm}, 20 \mathrm{~cm}, 30 \mathrm{~cm}$ e $40 \mathrm{~cm}$, para o lisímetro L2. Na Tabela 6, apresentam-se os resultados dos testes de aderência entre as simulações e as medições efetuadas para o período total de 100 dias.
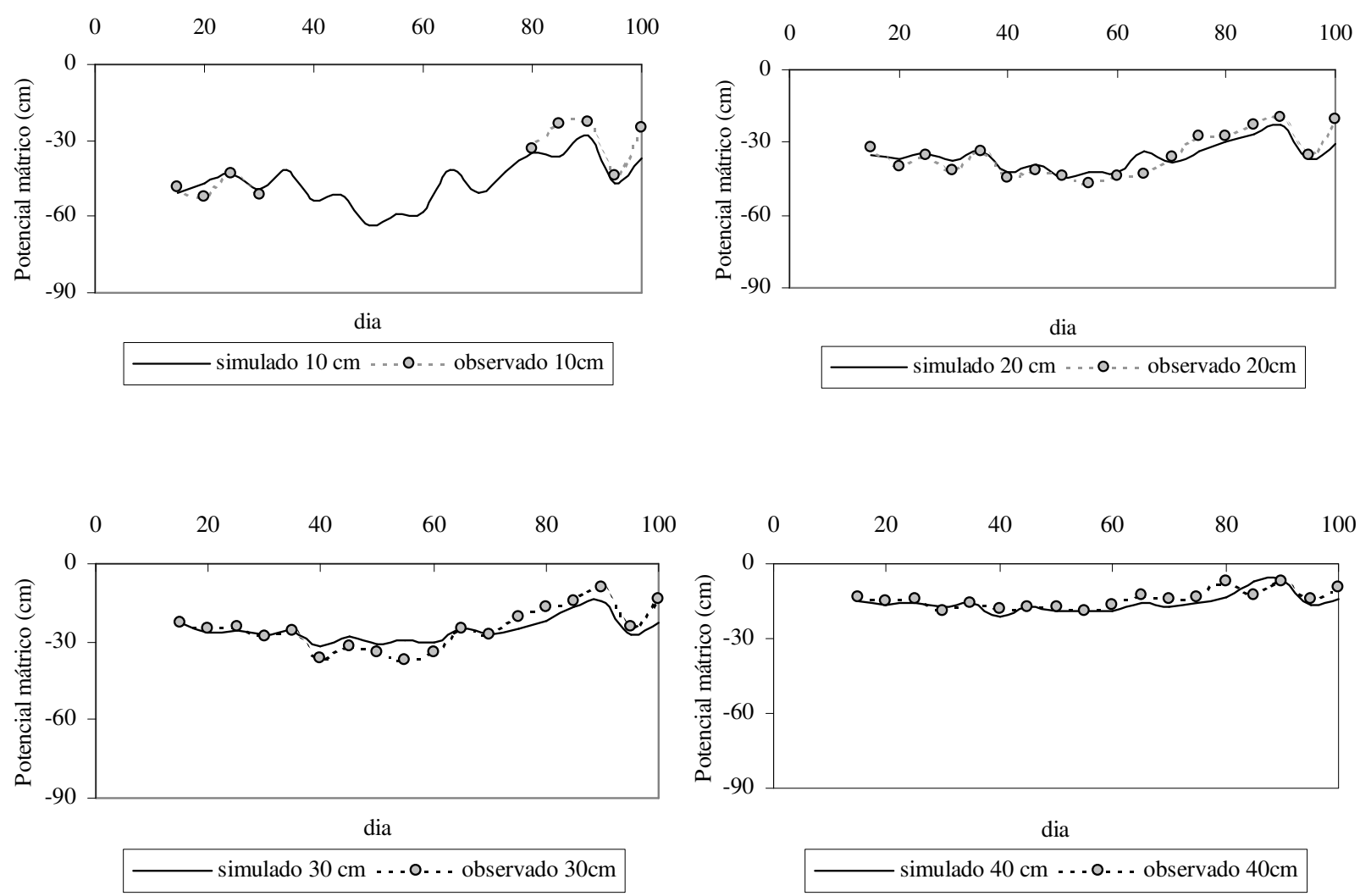

FIGURA 2. Comparação entre potenciais mátricos medidos e simulados para o lisímetro L2, em pentadas. Comparison between measured and simulated matric potentials for the L2 lysimeter by using five days average values.

As precisões das estimativas numéricas foram verificadas considerando o erro quadrado médio (EQM), bem como o coeficiente "D" de Willmott, conforme expressões apresentadas em ARBAT et al. (2003).

TABELA 6. Coeficiente de Willmott (D) e erro quadrático médio (EQM), por lisímetro, considerando potenciais medidos e simulados. Willmott coefficient (D) and mean squared error (EQM) for each lysimeter considering measured and simulated potentials.

\begin{tabular}{clllll}
\hline & & $10 \mathrm{~cm}$ & $20 \mathrm{~cm}$ & $30 \mathrm{~cm}$ & $40 \mathrm{~cm}$ \\
\hline \multirow{2}{*}{ L1 } & D & 38,27 & 83,73 & 83,75 & 67,63 \\
& EQM & 44,91 & 15,82 & 12,70 & 17,10 \\
\hline \multirow{2}{*}{ L2 } & D & 99,01 & 90,50 & 74,92 & 84,92 \\
& EQM & 14,65 & 12,35 & 24,49 & 19,68 \\
\hline \multirow{2}{*}{ L3 } & D & 34,44 & 97,71 & 57,27 & 78,65 \\
& EQM & 37,90 & 19,32 & 14,77 & 29,60 \\
\hline
\end{tabular}

De modo geral, verifica-se, na Tabela 6 , menor aderência entre as simulações e as medições de potenciais mátricos para a profundidade de $10 \mathrm{~cm}$. Essa baixa aderência pode estar associada a dificuldades operacionais de medir tensão a $10 \mathrm{~cm}$, tendo em vista possíveis problemas de 
isolamento da cápsula cerâmica, bem como às variações diurnas de potenciais mátricos, não considerados no valor observado dessa variável. Tais inconsistências para as camadas subsuperficiais também foram constatadas por GONÇALVES et al. (2006). Apenas o lisímetro L2 apresentou adequado ajuste, com coeficiente de Willmott superior a 90\%, embora apresentando algumas falhas nas medidas. A profundidade de $20 \mathrm{~cm}$, onde se situa a maior parte da zona de raízes, apresenta valores simulados consistentes às medidas efetuadas nos tensiômetros, para todos os lisímetros considerados.

$\mathrm{Na}$ Figura 3, apresentam-se as simulações de transporte de sais, tendo como saída a condutividade elétrica para as profundidades de 20;30 e $40 \mathrm{~cm}$, para os três lisímetros. As simulações tenderam a seguir a dinâmica de valores medidos, particularmente quando da ocorrência de umedecimentos bruscos. Os valores calibrados de dispersividade foram de $4 \mathrm{~cm}$, para a camada de 0 a $20 \mathrm{~cm} ; 3 \mathrm{~cm}$, para as camadas de 20 a $40 \mathrm{~cm}$ e 40 a $70 \mathrm{~cm}$; e $2 \mathrm{~cm}$ para a camada de 70 a $80 \mathrm{~cm}$. Podem-se notar algumas discrepâncias entre valores medidos e simulados para o modelo, embora a tendência de lixiviação, com os eventos chuvosos, esteja preservada em termos gerais.
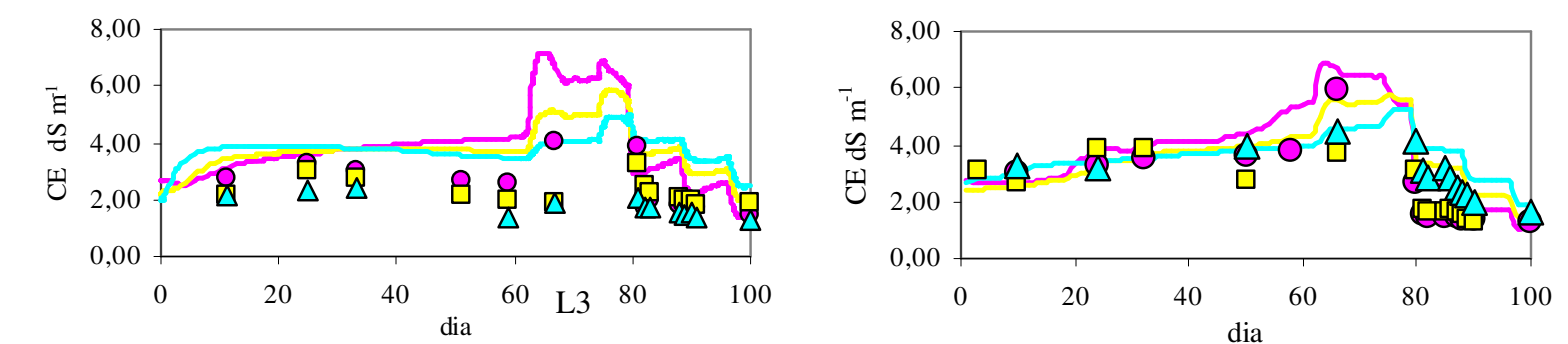

\begin{tabular}{|c|c|c|}
\hline CE $20 \mathrm{~cm}$ & CE $30 \mathrm{~cm}$ & $-\mathrm{CE} 40 \mathrm{~cm}$ \\
\hline CE obs $20 \mathrm{~cm}$ & $\square \quad \mathrm{CE}$ obs $30 \mathrm{~cm}$ & $\triangle$ CE obs $40 \mathrm{~cm}$ \\
\hline
\end{tabular}

\begin{tabular}{|c|c|c|}
\hline CE $20 \mathrm{~cm}$ & CE $30 \mathrm{~cm}$ & $-\mathrm{CE} 40 \mathrm{~cm}$ \\
\hline O CE obs $20 \mathrm{~cm}$ & $\square \quad \mathrm{CE}$ obs $30 \mathrm{~cm}$ & $\triangle$ CE obs $40 \mathrm{~cm}$ \\
\hline
\end{tabular}

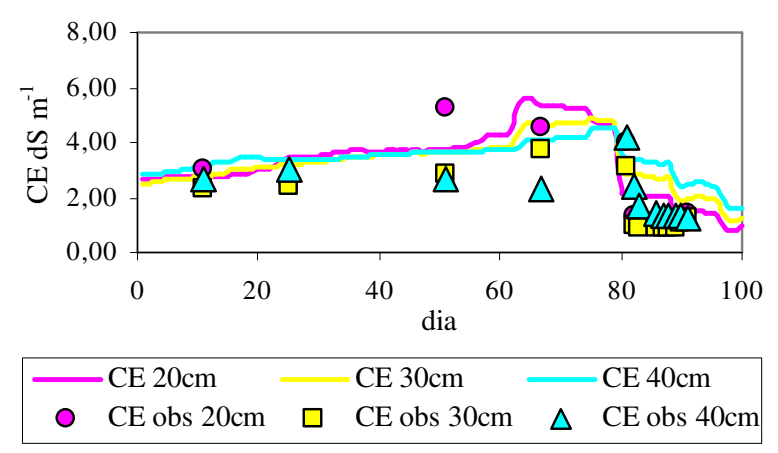

FIGURA 3. Valores de condutividade elétrica simulada e medida em campo, para os lisímetros L1, L2 e L3. Measured and simulated electrical conductivity values for each lysimeter L1, L2 and L3.

Nos lisímetros L2 e L3, pode-se notar maior aderência do modelo aos dados experimentais. Embora valores discrepantes tenham ocorrido, as simulações oferecem importantes informações no tocante à evolução da salinidade durante os experimentos de campo, podendo-se claramente observar a relevância das precipitações pluviométricas, as quais foram eficientes na lavagem do perfil. As condutividades elétricas médias da solução do solo ao final do ciclo de cultivo, observadas nos lisímetros, mesmo com frações de lixiviação inferiores à necessidade de lavagem, foram equivalentes e iguais a 1,56 ( $\pm 0,28) ; 1,44( \pm 0,25)$ e $1,32( \pm 0,11) \mathrm{dS} \mathrm{m}^{-1}$, respectivamente, enquanto as simulações computacionais produziram valores de $2,02( \pm 0,43) ; 1,49( \pm 0,40)$ e 1,82 $( \pm 0,43) \mathrm{dS} \mathrm{m}{ }^{-1}$ para os três lisímetros, respectivamente. 


\section{Simulação de cenários}

Considerando-se adequadas as calibrações e validações efetuadas, foram, então, realizadas simulações computacionais para as seguintes frações de lixiviação: $\mathrm{FL}=0 ; \mathrm{FL}=0,09 ; \mathrm{FL}=0,17$; $\mathrm{FL}=0,23 ; \mathrm{FL}=0,29 ; \mathrm{FL}=0,33$ como apresentados pelas Figuras 4; 5 e 6, de modo a investigar tanto o efeito de lixiviações completas quanto parciais.

O objetivo de tais análises foi refinar o valor da fração de lixiviação que otimizasse a aplicação hídrica, tanto no sentido agrícola quanto com referência à gestão dos recursos hídricos, particularmente de economia de água.

Simulou-se água de condutividade elétrica, em média, de $1,10 \mathrm{dS} \mathrm{m}{ }^{-1}$, e condutividade elétrica inicial da solução do solo igual aos valores medidos no início do experimento (Figuras 4; 5 e 6).
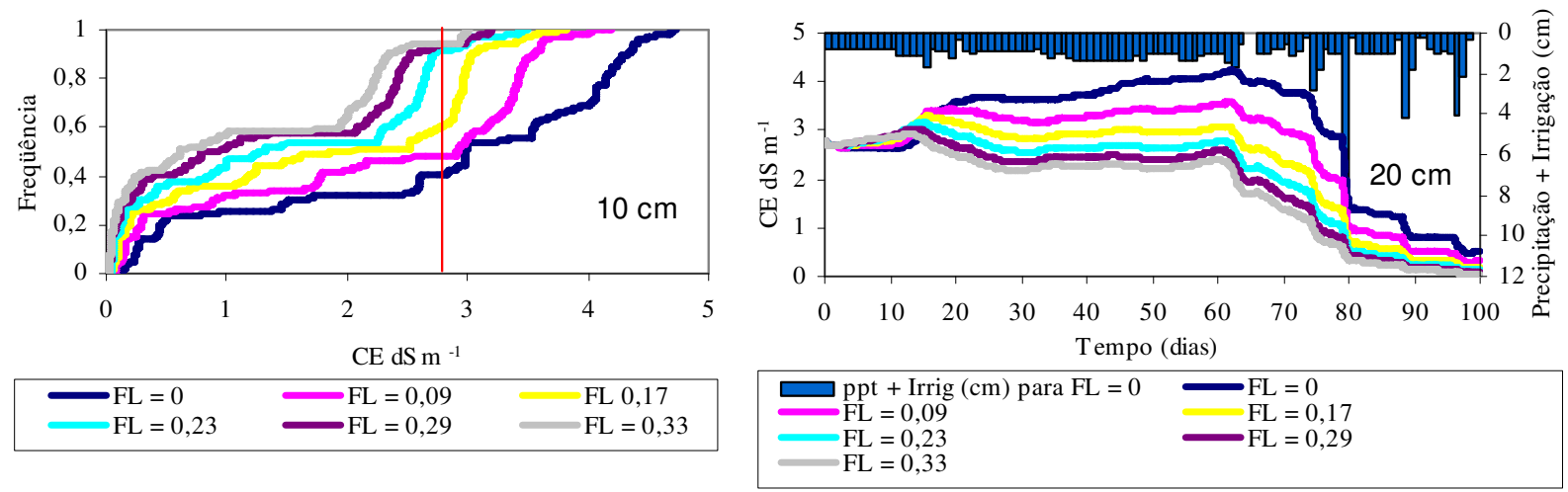

FIGURA 4. Distribuição de frequência acumulada e temporal da condutividade elétrica do extrato saturado para a profundidade de $10 \mathrm{~cm}$, de acordo com simulações, usando o modelo HYDRUS-1D para diferentes frações de lixiviação. Cumulative and temporal frequency distribution of the saturated extract electrical conductivity for the $\mathbf{1 0}$ cm depth, according to the simulations by using the HYDRUS-1D model for different leaching fractions.
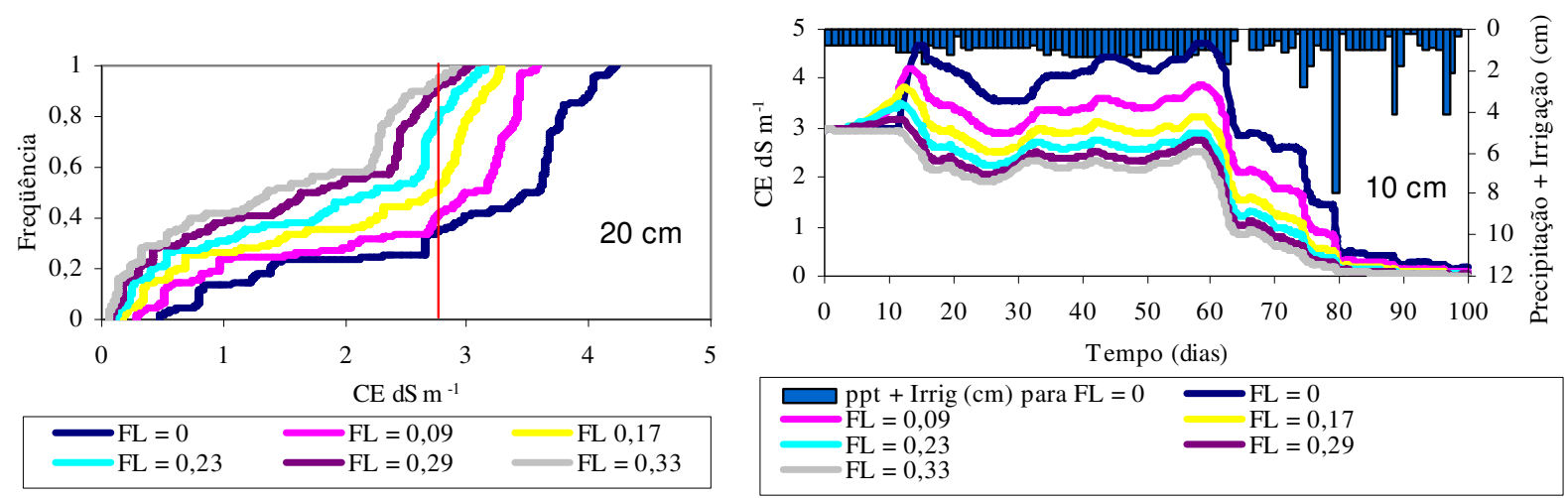

FIGURA 5. Distribuição de frequência acumulada e temporal da condutividade elétrica do extrato saturado para a profundidade de $20 \mathrm{~cm}$, de acordo com simulações, usando o modelo HYDRUS-1D para diferentes frações de lixiviação. Cumulative and temporal frequency distributions of the saturated extract electrical conductivity for the 20 cm depth, according to the simulations by using the HYDRUS-1D model for different leaching fractions. 

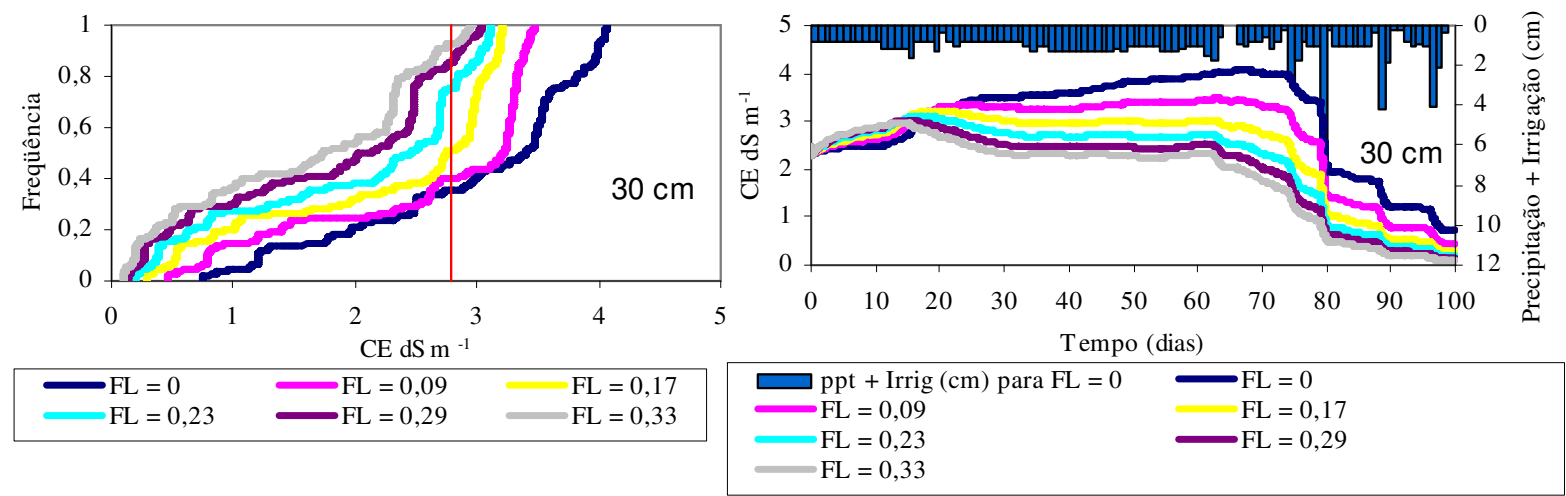

FIGURA 6. Distribuição de frequência acumulada e temporal da condutividade elétrica do extrato saturado para a profundidade de $30 \mathrm{~cm}$, de acordo com simulações, usando o modelo HYDRUS-1D para diferentes frações de lixiviação. Cumulative and temporal frequency distributions of the saturated extract electrical conductivity for the $\mathbf{3 0}$ cm depth, according to the simulations by using the HYDRUS-1D model for different leaching fractions.

Nos gráficos de frequência acumulada, apresentados nas Figuras 4 a 6, está destacada, a título de exemplo, a salinidade de 2,80 dS m $\mathrm{m}^{-1}$, considerada por AYERS \& WESTCOT (1999) como sendo a condutividade limite para rendimento potencial de $75 \%$, para a cultura da cenoura. Na Tabela 7, apresentam-se as frequências acumuladas de não excedência referentes às profundidades de 10; 20 e 30 cm, e as frequências percentuais, considerando as frações de lixiviação analisadas.

A condutividade elétrica média do extrato de saturação, para os cenários de lixiviação simulados, foi de $0,20( \pm 0,19) \mathrm{dS} \mathrm{m}^{-1}$ para a camada de 10 a $30 \mathrm{~cm}$, ao final do ciclo da cultura, conforme os valores exibidos nas Figuras 4; 5 e 6.

TABELA 7. Frequências acumuladas de não excedência $\mathrm{P} \%$ do valor limite de $C E=2,80 \mathrm{dS} \mathrm{m}^{-1}$. Non exceeding cumulative frequencies $P \%$ for the threshold $E C=2.80 \mathrm{dS} \mathrm{m}^{-1}$.

\begin{tabular}{ccccccc}
\hline \multirow{2}{*}{$\mathrm{Z}(\mathrm{cm})$} & \multicolumn{7}{c}{$\mathrm{FL}$} \\
\cline { 2 - 7 } & 0 & 0,09 & 0,17 & 0,23 & 0,29 & 0,33 \\
\hline 10 & 41 & 48 & 61 & 91 & 94 & 94 \\
20 & 35 & 42 & 54 & 82 & 92 & 96 \\
30 & 35 & 40 & 51 & 75 & 86 & 93 \\
\hline Média & 37,0 & 43,3 & 55,3 & 82,7 & 90,7 & 94,3 \\
\hline
\end{tabular}

Considerando que, no período, ocorreram $340 \mathrm{~mm}$ de precipitação pluviométrica, frente à necessidade hídrica (assumida potencial) de $784,36 \mathrm{~mm}$, deve-se investigar qual quantidade de água é necessária para promover tais lixiviações. Tais valores de lâmina necessária estão apresentados na Tabela 8.

TABELA 8. Lâminas totais irrigadas para cada fração de lixiviação. Total irrigated depths for each leaching fraction.

\begin{tabular}{ccrrrrr}
\hline FL & 0 & 0,09 & 0,17 & 0,23 & 0,29 & 0,33 \\
\hline $\mathrm{I}(\mathrm{cm})$ & 44,43 & 48,87 & 53,32 & 57,77 & 62,21 & 66,65 \\
\hline
\end{tabular}

Na Figura 7, apresentam-se os valores médios das frequências acumuladas de não excedência do valor limite, bem como a lâmina de água de irrigação necessária à lixiviação pretendida. Pode-se observar que, para o cenário de chuva em discussão, há ganho significativo em se aumentar o FL de 
0,09 para 0,17 (região da função matemática com maior crescimento). A partir de 0,17 , acréscimos de lâmina (lineares) produzem incremento de percentual de não excedência igualmente lineares. A escolha do coeficiente ótimo dependeria de fatores econômicos, ligados ao custo agregado a cada milímetro de água alocada, bem como do benefício associado ao incremento da produção, que depende do valor de mercado da cultura. De qualquer modo, há elementos técnicos para sugerir frações de lixiviação FL na faixa entre 0,09 e 0,17. Com efeito, para a fração de lixiviação igual ou superior a 0,17, a função de probabilidade P (Figura 7) apresenta um ponto de inflexão e assim se distancia do valor ótimo.

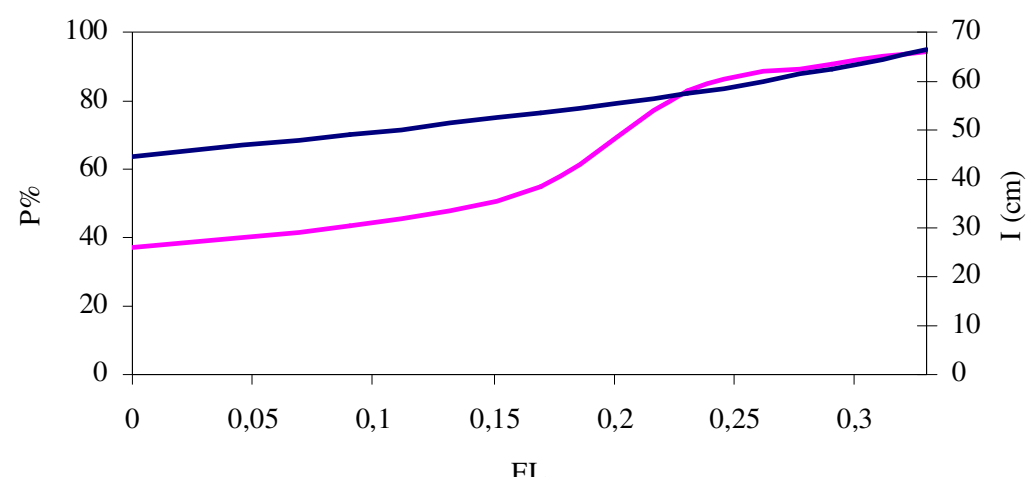

FL

— Lâmina de irrigação (irrig) — função de probabilidade P

FIGURA 7. Lâminas de irrigação (Irrig) e percentuais de não excedência do valor limite (P) para cada fração de lixiviação testada. Irrigated depths (Irrig) and non-exceeding percentages for threshold percentage (P), for each tested leaching depth.

Caso se desejasse otimizar a produção (para rendimento potencial de $75 \%$ ), a fração de lixiviação, na faixa de 0,09 a 0,17 , poderia ser escolhida.

\section{CONCLUSÕES}

As simulações realizadas com o modelo HYDRUS-1D apresentaram ajustes adequados aos dados medidos.

Com base na montagem experimental e nos estudos de caso considerados, foi possível calibrar o modelo HYDRUS, que pode ser utilizado como ferramenta para a simulação de transferência de sais em meio irrigado, na área estudada.

Os valores adotados para a lixiviação, considerando lixiviação parcial nos lisímetros, não comprometeram a produção da cenoura, podendo-se aceitar que a cultura se desenvolveu conforme as características locais de qualidade de água para irrigação e clima.

Os cenários produzidos com diferentes frações de lixiviação indicam que a produção pode ser otimizada para uma fração de lixiviação entre 0,17 e 0,23.

Para todos os cenários de fração de lixiviação considerados, observou-se, ao final do ciclo da cultura, condutividade elétrica média do extrato saturado inferior a $0,74 \mathrm{dS} \mathrm{m}^{-1}$.

Os resultados obtidos indicam a viabilidade de se utilizar lixiviação parcial de sais no local de estudo, para as condições analisadas, desde que chuvas complementem a lavagem dos sais.

\section{REFERÊNCIAS}

ABOUKHALED, A.; ALFARO, A.; SMITH, M. Lysimeters. Rome: FAO, 1982. 68 p. (Irrigation and Drainage, 39). 
ARBAT, G.; BARRAGÁN, J.; PUIG, J.; POCH, R.; CARTAGENA R.F. Evaluación de los modelos numéricos de flujo de agua en el suelo HYDRUS-2D y SIMDAS en riego localizado. In: ÁLVAREZ-BENEDÍ, J.; MARINERO, P. Estudios de la zona no saturada del suelo. Valladolid: Instituto Tecnológico Agrario de Castilla y León (I.T.A.), 2003. v.6, p.279-288. (Actas de las VI Jornadas sobre Investigación de la Zona no Saturada del Suelo).

AUDRY, P.; SUASSUNA, J. A salinidade das águas disponíveis para pequena irrigação no sertão nordestino. Caracterização. Variação sazonal. Limitação de uso. Recife: Conselho Nacional de Desenvolvimento Científico e Tecnológico - CNPq 1995. 128 p.

AYERS, R.S.; WESTCOT, D.W. A qualidade de água na agricultura. 2.ed. Campina Grande: UFPB, 1999. 153 p. (FAO Irrigação e Drenagem, 29 revisado 1).

BASTOS, D.C.O. Manejo da salinidade em irrigação localizada: análise da alternativa de lixiviação incompleta. 2004. 57 f. Dissertação (Mestrado em Ciência do Solo) - Universidade Federal Rural de Pernambuco, Recife, 2004.

DOORENBOS, J.; KASSAM, A.H. Efectos del água en al rendimiento de los cultivos. Roma: FAO, 1979. 212 p (Riego y Drenage, 33).

EMBRAPA. EMPRESA BRASILEIRA DE PESQUISA AGROPECUÁRIA. Manual de métodos de análise de solo. 2.ed. Rio de Janeiro, 1997. 212 p.

FEDDES, R.A.; KOVALIK, P.J.; ZARADNY, H. Simulation of field water use and crop yield. New York: John Wiley, 1978.188 p.

FORKUTSA, I. Modeling water and salt dynamics under irrigated cotton with shallow groundwater in the Khorezm region of Uzbekistan. 2006. $158 \mathrm{f}$. Thesis (Doctoral) - Faculty of Agriculture, Center for Development Research - ZEF, University of Bonn, Bonn, 2006.

GENUCHTEN, M.T. Van. A closed-form equation for predicting the hydraulic conductivity of unsaturated soils. Soil Science Society of America Journal, Madison, v.44, n.5, p.892-898, 1980.

GENUCHTEN, M.T. Van. A numerical model for water and solute movement in and below the root zone. Riverside: US Salinity Laboratory, 1987. 221 p. (Research Report)

GONÇALVES, M.C.; ŠIMU゚NEK, J.; RAMOS T.B.; MARTINS J.C.; NEVES, M.J.; PIRES F.P. Multicomponent solute transport in soil lysimeters irrigated with waters of different quality. Water Resources Research. Disponível em: <http://dsc.doi.org>. DOI: 10.1029/2005WR004802, 2006.

MAAS, E.V.; HOFFMAN, G.J. Crop salt tolerance: current assessment. Journal of Irrigation and Drainage Div, ASCE, New York, v.103, p.115-134, 1977.

MONTENEGRO, S.M.G.L.; MACKAY, R.; MONTENEGRO, A.A.A. Análise estocástica de fluxo e transporte em solos não saturados para avaliação de risco de salinização. Revista Brasileira de Recursos Hídricos, Porto Alegre, v.7, n.2, p.17-34, 2002.

MONTENEGRO, S.M.G.L.; MONTENEGRO, A.A.A.; RIBEIRO, M.R.; CORREA, M.M.; ALMEIDA, T.A.; LINS, F.V. Análise da variabilidade espacial da salinidade em área irrigada e do nível d' água em aluvião sob uso agrícola na região semiárida do Nordeste brasileiro. In: SIMPÓSIO BRASILEIRO DE RECURSOS HÍDRICOS, 14., 2001, Aracaju. Anais... Aracaju: Associação Brasileira de Recursos Hídricos - ABRH, 2001. 1 CD-ROM.

MUALEM, Y.A. A new model for predicting the hydraulic conductivity of unsaturated porous media. Water Resources Research, Washington, v.12, n.3, p. 512-522, 1976.

NEVES, R.; CHAMBEL-LEITÃO, P.; LEITÃO, P.C. Modelação numérica da circulação da água no solo, o modelo mohid. Pedologia, Oeiras, v.28, n.1, p.46-55, 2000.

RHOADES, J. Drainage for salinity control. In: SHILFGAARDE J. Van (Ed.). Drainage for Agriculture. Madison: American Society of Agronomy, 1974. p.433-462. 
RIBEIRO, M.R.; CORRÊA, M.M. Levantamento detalhado de solos da Fazenda Nossa Senhora do Rosário. In: MONTENEGRO, A.A.A. Diagnóstico e controle da salinização em aluviões com pequena agricultura irrigada. Recife: UFRPE, 2001. 34 p. (Relatório)

SANTIAGO, F. dos S. ; MONTENEGRO, A.A.A. ; MONTENEGRO, S.M.G.L . Avaliação de parâmetros hidráulicos e manejo da irrigação por microaspersão em área de assentamento.

Engenharia Agrícola, Jaboticabal, v.24, n.3, p.632-643, 2004.

SANTOS, F.X. Evapotranspiração de cultura e influência de diferentes lâminas e frequências de irrigação no crescimento e produtividade da cultura da cenoura. 2008. 115 f. Tese (Doutorado em Ciência do Solo) - Universidade Federal Rural de Pernambuco, Recife, 2008.

SANTOS, F.X.; RODRIGUES, J.J.V.; MONTENEGRO, A.A.A.; MOURA, R.F. Desempenho de lisímetro de pesagem hidráulica de baixo custo no semiárido nordestino. Engenharia Agrícola, Jaboticabal, v.28, n.1, p.115-124, 2008.

SILVA, E.F.F.; ANTI, G.R.; CARMELO, Q.A.C.; DUARTE, S.N. Extratores de cápsulas porosas e o monitoramento da condutividade elétrica e o teor de potássio na solução de um solo. Scientia Agrícola, Piracicaba, v.57, n.4, p.785-789, 2000.

SILVA, T.J.A.; MONTENEGRO, A.A.; RODRIGUES, J.J.V.; BONFIM, E.M.S. Aplicação de lisímetro de pesagem hidráulica na determinação da evapotranspiração de referência, em Petrolina PE. Engenharia Agrícola, Jaboticabal, v.23, n.3, p.511-520, 2003.

ŠIMŮNEK, J.; SEJNA, M.; GENUCHTEN, M.T. Van. The HYDRUS-1D software package for simulating the one-dimensional movement of water, heat, and multiple solutes in variably-saturated media. Version 3.0, IGWMC-TPS-70. Golden: International Ground Water Modeling Center, Colorado School of Mines, 2005. 270 p.

SUOJALA, T. Growth of and partitioning between shoot and storage root of carrot in a northern climate. Agricultural and Food Science in Finland, Piikkiö, v.9, n.1, p.49-59, 2000.

VANCLOOSTER, M.; VIANE, P.; DIELS, J.; CHRISTIAENS, K. WAVE: A mathematical model for simulating water and agrochemicals in the soil and vadose environment. Reference and User's Manual (release 2.0). Belgium: Institute for Land and Water Management, Katholieke Universiteit Leuven, 1994. $145 \mathrm{p}$. 